\title{
Evaluation of Partially Described Multiattribute Options
}

\author{
J. Frank Yates, Carolyn M. Jagacinski, and \\ MARK D. FABER
}

\section{The University of Michigan}

\begin{abstract}
Thirty-five subjects rated the satisfactoriness of two sets of 125 profiles of hypothetical university courses. The profiles in one set were all complete, containing indicators of the state of each course on each of four attribute dimensions. Fifty of the profiles in the second set were partial representations of profiles from the first set, reporting that the level of one of the attributes was "unknown." The other 75 profiles were identical to the remaining profiles in the first set. Results indicated that subjects tended to devalue the partial profiles relative to the complete profiles $(p<.01)$. Regression equations characterizing evaluations of the 75 complete profiles presented to subjects in both replications were derived. Regression weights for the dimension that was omitted in partial profiles tended to be larger in the replication in which complete profiles were accompanied by partial profiles. The results are discussed in terms of uncertainty avoidance and attention and cognitive strain effects on evaluation policies.
\end{abstract}

There is a great deal of current interest in how people evaluate and choose among options characterized by several relevant attributes rather than just one (Slovic, Fischoff, \& Lichtenstein, 1977; Keeney \& Raiffa, 1976). It is clear that the typical shopper's attraction to a new coffee maker depends on more than its price. Similarly, one's preferences among various potential careers likely rest on more than their income potentials. Research on multiattribute options has focused on a variety of specific issues, including questions about which attributes the subject is likely to take into account, how judgments of an option's attributes are synthesized into an overall evaluation, and how the subject ultimately comes to make a choice among a set of available alternatives.

The present research addresses a problem that surely arises quite often in practical evaluation and decision situations, yet has curiously received little previous attention. A subject might identify attribute dimensions that

This research was supported by Grant MH 27000 from the National Institute of Mental Health. Preliminary research was made possible by a grant from the University of Michigan's Rackham School of Graduate Studies. It is our pleasure to acknowledge the valuable contributions of Jim Tavrazich and Zvi Aran to the conduct of the experiment reported. We are also grateful for the helpful suggestions offered by Paul Green.

Requests for reprints should be sent to J. Frank Yates, Department of Psychology, 136 Perry Building, University of Michigan, Ann Arbor, Michigan 48109. 
are significant to his/her evaluations of a class of objects, but information about the states of available options on all those relevant dimensions may not be accessible, i.e., options can sometimes be partially described. For example, a list summarizing the major characteristics of applicants for graduate fellowships is likely to include candidates whose grade point averages or test scores are missing. Or, in the process of reviewing position descriptions and actual employment offers, a promotion-conscious job seeker may encounter employers whose promotion policies are simply unavailable. Swinth's (1976) study of the processes business school graduates used in deciding among job offers provides a concrete illustration of the incidence of such problems. As part of the study's procedure, the graduates identified the dimensions of potential employment positions that were important to their deliberations. The subjects were also required to report the state of each position on those personally significant attribute dimensions. In 10\% of all cases, the subjects had to report "Don't know."

Two specific questions about the partial description of multiattribute options were confronted in the present investigation. First, are subjects' evaluations of partially described options different from their evaluations of completely described options? Second, does the presence of a substantial number of partially described options embedded within an array of completely described options affect subjects' policies for evaluating those completely described options?

Despite the absence of previous research directly addressing the partial description problem, plausible arguments in favor of various alternative hypotheses about the issues can be derived from tangentially related investigations. Consider first what may be called the discrepancy question, the issue concerning the evaluation of partially described as compared to completely described options. In Swinth's (1976) study of business students' job decisions, when subjects reported that they did not know the state of a potential position on a given attribute dimension, the investigator treated such responses as if the attribute state was at a desirable level. Implicitly, the claim is made that the subjects themselves responded to partially described outcomes favorably. Unfortunately, Swinth does not offer a rationale for this claim. Moreover, his data analysis does not permit a conclusion to be drawn about empirical support for the position. Slovic and MacPhillamy (1974) convey a suggestion by Castellan that when a partially described option is encountered, the subject judges the option as though its status on the missing dimensions is "average." AIthough no theoretical basis for this suggestion was elaborated, it certainly seems intuitively reasonable. Slovic and MacPhillamy did not directly test the average status hypothesis. However, the introspective comments of 
several subjects in their study of a related phenomenon were corroborative.

The remaining hypothesis, that partially described options are devalued relative to their completely described counterparts, has supporting arguments, too. A partially described option can be viewed as an uncertain alternative in the same way that, say, a game of chance can. For concreteness, think of a job that is completely described except for the working locale; it might be anywhere from Pleasant City to Dreary Village. The job, if it happens to be located in Pleasant City, is clearly a better opportunity than a job that is identical, except located in Dreary Village. The partially described job offer is effectively a gamble whose outcome can be any number of distinct jobs which might differ in terms of their satisfactoriness to the job seeker. Given that he/she does not know which of those jobs will actually materialize, how should the person evaluate the original, partially described opportunity? Obviously, that evaluation should have something to do with the person's judgment of how likely it is that those various, distinct potential jobs will indeed eventuate.

A general conclusion that can be drawn from the decision making literature is that people avoid uncertainty (cf. Lee, 1971). It appears that most people are "risk averse" in the sense that when given the choice between, for example, $\$ 1.00$ for sure and a gamble offering $\$ 2.00$ if a fair coin toss results in a "Head" and nothing otherwise, they generally choose the guaranteed $\$ 1.00$. Such risk aversion is accommodated in decision analyses by concave utility functions (Raiffa, 1968).

There are other forms of uncertainty avoidance that seem to transcend simple risk aversion. A person may realize that the potential consequences of a decision alternative are not assured, yet have either firmly or weakly grounded bases for judging the likelihood of those consequences actually occurring. If the person has very solidly based likelihood opinions, the level of uncertainty involved in the situation is sometimes characterized as "risky" (Luce \& Raiffa, 1957, p. 12). The previously described bet contingent on a coin toss is an example of a risky decision alternative. If the person has no basis whatever for judging the likelihood of the outcomes of an event, the condition of pure "ignorance" is said to prevail (Coombs, Dawes, \& Tversky, 1970, p. 116). A wager dependent on whether the second place finisher in the 1980 Olympic decathlon prefers the color red to the color blue involves a level of uncertainty approaching ignorance. Most realistic choice situations involve levels of uncertainty intermediate between risk and pure ignorance. Ellsberg (1961) has coined the term "ambiguity" for these other levels of uncertainty. There have been numerous demonstrations that people avoid ambiguously uncertain options (Ellsberg, 1961; Becker \& Brownson, 1964; Yates \& 
Zukowski, 1976). Moreover, it is not difficult to show that their avoidance of such options implies an aversion that is over and above any aversion the subjects might have for the simple risk implicit in the options; subjective expected utility models cannot account for the preferences, regardless of utility function shapes.

The degree of uncertainty experienced by a subject confronted with a partially described option may be at any level. The evaluator's judgment as to the state of the option on the nondescribed attribute dimensions may be based on very rich or very sparse information. At any rate, generalization of the oft-observed phenomena of risk aversion and ambiguity avoidance suggests that partially described options should be evaluated by subjects less favorably than completely described ones.

What about the context effects of partially described options on policies for evaluating accompanying completely described options? Again, there are plausible arguments and previous evidence consistent with conflicting conclusions. Slovic and MacPhillamy (1974) report experiments suggesting that when subjects make pairwise comparative judgments of options, a given attribute dimension is weighted more heavily if it is common to both members of a pair than if it is unique. The explanation offered for this result is that comparisons along common dimensions demand less "cognitive strain" than comparisons that require the subject to make evaluations across dimensions, as is necessary if one wishes to take a unique dimension into account. The present investigation is concerned with a somewhat different situation from that studied by Slovic and MacPhillamy. The subject is presented with a series of option displays that are to be given individual rather than comparative evaluations. However, if the notion that subjects make evaluations in a fashion that reduces cognitive strain has generality, then their judgments of the individual members of a sequence of displays should be spontaneously influenced by one another, resulting in an effect similar to that observed by Slovic and MacPhillamy. That is, an attribute dimension that is omitted in partially described options should be weighted less heavily in accompanying completely described options since implicit comparisons between contiguous partially and completely described alternatives cannot be made with respect to that dimension.

Ample evidence (e.g., Summers, Taliaferro, \& Fletcher, 1970) indicates that when people make judgments of large numbers of multiattribute objects, their judgments are influenced substantially by very few of those attributes. This tendency reveals itself in the regression equations used for representing judgment policies. Such equations usually have nontrivial weights for only a small number of the attribute dimensions available. This result conflicts with subjects' verbally expressed judgment policies. Generally, subjects report that their judgments are affected by more attri- 
bute dimensions than the regression analyses indicate they are. One plausible interpretation of the discrepancy between intended and actual judgment policies is that although the subject wishes to take many attributes into account, his/her attention is successfully captured by only a few. If such an explanation has credence, then judgment policies should be susceptible to a variety of manipulations that affect attention to particular dimensions. Specifically, if an array of completely described multiattribute options is interspersed with occasional partially described options, attention should be drawn to the feature that distinguishes those partially described options. That is, policies for evaluating completely described options accompanied by partially described ones should appear to place more emphasis on the occasionally missing dimension than otherwise.

The experiment conducted in the present investigation sought to provide direct tests of the issues described above. The basic approach was as follows. Subjects evaluated four attribute descriptions of a series of options on two occasions. On one occasion the descriptions were complete, indicating the status of each option on all four attributes. The same options were described on another occasion, on which a subset of the descriptions were partial, omitting indicators of the status of the options on one particular dimension. In view of the weight of prior evidence concerning people's avoidance of uncertainty, it was hypothesized that subjects would devalue options when they were partially rather than completely described. The alternative underlying processes that might explain context effects of partially described options imply opposite predictions. Nevertheless, those processes are not inherently incompatible with each other; subjects might simultaneously maintain tendencies to adopt evaluation strategies that reduce cognitive strain and to attend to attributes that are "unusual" in that they are occasionally omitted. From this perspective, the context issue reduces to a question of the relative magnitudes of the conflicting effects. Slovic and MacPhillamy (1974) have already presented evidence of the existence of the cognitive strain effect. Payne (1976) has reported results consistent with such an effect, also. Is there reason to believe that the attention effect exists in sufficient strength to offset or even dominate the cognitive strain effect?

\section{METHOD}

\section{Subjects}

Thirty-five students (18 males and 17 females) at the University of Michigan served as subjects. They were recruited from the Human Performance Center's paid volunteer subject pool. Each subject was paid $\$ 5.00$ for participating in the study. Subjects participated in two sessions, lasting a total of approximately $2 \mathrm{hr}$. 


\section{Stimuli}

Stimuli consisted of "profiles" of hypothetical, nonrequired, university courses which varied along four attribute dimensions. The dimensions, displayed left to right, were: (1) Interest, "How interesting you find the subject matter;"' (2) Level of Instruction, "Degree to which the instructor teaches near the students' backgrounds and ability levels;' (3) Grade Leniency, "How lenient the instructor is in assigning grades;" and (4) Work Load, "Amount of work required for the credit received." These particular dimensions or "factors" as they were described to the subjects, were used for two reasons. First, preliminary testing indicated that students generally consider them to be very pertinent to the attractiveness of courses. Second, the dimensions seem to be intuitively independent of one another, in the ecological and value senses of the term.

It was necessary to establish appropriate anchors for the factors describing courses. Each subject was told to "create your own scale ranging from the best level to the worst level for each of the four factors." The "best" level of a factor was defined as "that level of the factor (a) you can reasonably expect some elective course to have and (b) would be most attractive to you-not necessarily anyone else." The "worst" level of a factor was defined analogously. To make certain that each subject had a concrete interpretation of each dimension, he/she was required to write down real or imaginary instances of courses or instructors exemplifying his/her notions of the best and worst levels of each factor. This anchoring procedure was intended to ensure that the scales used in describing profiles to subjects would be unidirectional and oriented the same way across factors.

A set of 125 sequences of four digits was generated. Each position in a sequence corresponded to one of the dimensions along which course profiles were varied. Digits 1-5 were used in the sequences. Thus, courses could assume any one of five levels on each factor, ranging from best (5) to worst (1). The 125 digits for each dimension approximated a normal distribution with mean 3 and standard deviation 1. The four quasinormal distributions of digits were essentially uncorrelated.

Two types of profiles for display to subjects were constructed. Each complete profile consisted of an array of "Xs" marked on four vertical Likert-type scales corresponding to particular course dimensions. The marks could appear at any one of five equally spaced points along each scale. The top of each scale stood for the best level of the respective factor, while the bottom represented the worst level. Partial profiles were constructed the same as complete profiles except for the Level of Instruction dimension. Rather than an " $X$ ' along the Level of Instruction scale, partial profiles contained a centered message that the level of instruction of the course was "Unknown." 
Two sets of 125 profiles were constructed. Replication A profiles were all complete, based on the 125 sequences of four quasinormally distributed digits described above. Seventy-five of the Replication B profiles were identical to a subset of the Replication A profiles. The other 50 Replication B profiles were partial, being the same as the remaining Replication A profiles except, of course, for the omission of the Level of Instruction indicator. The profiles of a given replication were presented to the subject in a randomized order.

\section{Apparatus}

Profiles were displayed to subjects on $30.5-\mathrm{cm}$ diagonal Ball Miratell cathode ray tubes. As many as three subjects could be run simultaneously and independently on separate tubes contained in semienclosed booths. Each profile display also contained a horizontal Likert-type scale via which the subject could indicate the degree of satisfaction he/she would anticipate from taking the course represented by the profile.

Each subject was provided with a response button panel to control the presentation of profiles and to record his/her anticipated satisfaction with the courses represented by the profiles. The satisfaction scale accompanying a given profile contained an arrow which could point to any one of 51 equally spaced positions along the scale. Each trial began with this cursor located at the middle of the satisfaction scale. The control panel contained buttons that allowed the subject to move the cursor back and forth along the scale as often as heishe desired. When the cursor was located at a point corresponding to the subject's anticipated degree of satisfaction, he/she could have this judgment recorded by pushing a rating response button on the panel. Recording of a satisfaction rating simultaneously resulted in the display of the next profile in the sequence. The apparatus also included a button which allowed the subject to repeat a trial in the event he/she accidentally depressed the rating response button. The entire apparatus was under the control of an IBM 1800 data processing system.

\section{Procedure}

The two sessions in which each subject participated were approximately 1 week apart. During the first session the subject was told that the study was "concerned with the influence of certain factors on how satisfied students are with elective courses." After the four course dimensions were defined for the subject, he/she was required to establish his/her anchors for these dimensions as described above. The subject was shown illustrative profiles, including a partial profile with an unknown level of instruction. That illustration was accompanied by the statement, "While the course certainly will be taught at some level of instruction, information about that factor is simply unavailable." The subject then rated two 
practice profiles using paper and pencil. After it was clear that the subject understood the basic routine, he/she was instructed in how to carry out the procedure using the computer-controlled apparatus. At all times the response sheet containing the subject's factor anchors was in view. Following the rating of five practice profiles, the subject rated the 125 profiles appropriate for his/her assigned condition .

The second session attended by each subject proceeded according to essentially the same routine as the first. The subject was reminded that the study was concerned with how certain factors influenced satisfaction with elective courses. He/she was also told, "Since we need a large amount of information to understand exactly how those factors affect students' satisfaction with courses, I'd like to have you simply rate another set of course profiles." After a review of the procedures, the subject rated his/her second group of 125 profiles.

Approximately half the subjects rated the Replication A profiles during their first sessions and Replication B profiles during their second sessions. The remaining subjects were exposed to the profile sets in the opposite order.

\section{RESULTS}

The mean responses of subjects who were exposed to the Replication A and $B$ profiles in opposite orders were compared. Since the comparisons revealed no order effects, the subsequent analyses were performed across all subjects, ignoring profile set presentation order. To test the general consistency of subjects' responses from one session to the next, correlations between responses to Replication A and B complete profiles were computed. These correlations ranged from .702 to .962 , with a mean value of .834 .

\section{Discrepancy Effects}

Each partial profile in the Replication B set was paired with the Replication A profile identical to it except for the Level of Instruction description. The differences in the satisfaction ratings given such pairs of profiles were computed for each subject. There were 11 cases in which the Level of Instruction factor for the complete profile was high (scale value 4 or 5), 23 instances in which the factor was intermediate (scale value 3), and 16 cases in which Level of Instruction was low (scale value 1 or 2). Three discrepancy scores were computed for each subject. The subject's high discrepancy score consisted of the mean difference between the satisfaction ratings for the 11 pairs of complete and partial profiles with high Level of Instruction scale values. Intermediate and low discrepancy scores were defined similarly. Table 1 displays the mean discrepancy scores across all subjects.

All three mean discrepancy scores were compared to zero. The most 
TABLE 1

Complete-Partial Profile Satisfaction Discrepancy Scores"

\begin{tabular}{lcccc}
\hline $\begin{array}{c}\text { Complete profile } \\
\text { Level } \\
\text { of Instruction }\end{array}$ & $\begin{array}{c}\text { Number } \\
\text { of } \\
\text { cases }\end{array}$ & $\begin{array}{c}\text { Mean } \\
\text { discrepancy } \\
\text { level }\end{array}$ & $t(34)$ & $p$ \\
\hline High & 11 & 5.439 & 8.614 & $<.001$ \\
Intermediate & 23 & 1.308 & 2.883 & $<.01$ \\
Low & 16 & -3.802 & -7.218 & $<.001$ \\
\hline
\end{tabular}

" Discrepancy score $=\Sigma$ (complete profile rating - partial profile rating)/number of cases.

important comparison involves the intermediate discrepancy scores. The data indicate that omission of the Level of Instruction description results in a lower satisfaction rating than when the original factor scale value was intermediate $(p<.01)$. This result conflicts with the hypothesis that subjects behave as if the status of options on missing dimensions is "average." Low discrepancy scores were informative, also. They showed that subjects tended to evaluate partially described outcomes more highly than their paired completely described outcomes when the originally displayed Level of Instruction was low $(p<.001)$. Not surprisingly, when a completely described profile was high on the Level of Instruction factor, omitting the Level of Instruction description generally resulted in a lower satisfaction rating $(p<.001)$. In sum, these results imply that subjects are inclined to devalue partially described options. They seem to respond to such options as if their status were below par on the dimension that is not described. Nevertheless, subjects do not appear to adopt the extremely pessimistic stance of "assuming the worst," i.e., evaluating partially de-

TABLE 2

Meax Coefficients of Determination ano Regression Coefficients for Complete Profile Evallation Policy Equations in Complete and Partial Profile Contexts

\begin{tabular}{lccccc}
\hline & \multicolumn{4}{c}{ Mean factor regression coefficients } \\
\cline { 3 - 6 } \multicolumn{1}{c}{ Profile context block } & Mean $R^{2}$ & Interest & $\begin{array}{c}\text { Level of } \\
\text { Instruction }\end{array}$ & $\begin{array}{c}\text { Grade } \\
\text { Leniency }\end{array}$ & $\begin{array}{c}\text { Work } \\
\text { Load }\end{array}$ \\
\hline Complete & .814 & .666 & .368 & .288 & .208 \\
Partial & .816 & .653 & .400 & .310 & .206 \\
$t(34)^{\prime \prime}$ & -.120 & - & -1.816 & - & - \\
$p$ & $\mathrm{~ns}$ & - & $<.08^{\prime \prime}$ & - & - \\
\hline
\end{tabular}

Note. Only the test of Level of Instruction regression weights is pertinent to the study. Other regression weight comparisons were, nevertheless, nonsignificant.

"Repeated measures.

"Two-tailed test. 
scribed options as though undescribed dimensions were at their lowest levels.

\section{Context Effects}

Two regression equations for predicting satisfaction ratings from profile scale values were derived for each subject. One equation was derived via responses to the 75 complete profiles in Replication B; the other equation was based on the same 75 profiles, but as presented in Replication A along with other complete profiles rather than partial profiles. Table 2 displays for both equations the mean coefficients of determination and standardized regression coefficients (beta weights) for all the course factors.

On the average, the two equations were equally effective in predicting subjects' satisfaction ratings. Evaluation policy equations did not change drastically when complete profiles were presented in the context of partial rather than complete profiles. However, consistent with the hypothesis that attention effects might counteract or dominate cognitive strain effects, there was an inclination for subjects to attach more significance to the Level of Instruction factor in the partial profile context. Regression weights for that factor tended to be higher in the partial profile condition than in the complete profile condition ( $p<.08$, two-tailed test).

\section{DISCUSSION}

The results of the study suggest that incomplete knowledge of the characteristics of a multiattribute option is indeed likely to influence a person's evaluation of that option as well as his/her evaluation of other options presented along with it. There should be a bias to devalue the option that is ill described. Moreover, even among well-described options, attention will be drawn to the features that were unspecified in the partially described alternative.

The above conclusions are those that are consistent with the data obtained in the present investigation. They should be accepted, of course, in view of the limitations of the study. Future research should be directed toward establishing the reliability and generality of the results. One problem of generality concerns the use of artificial displays of fictitious outcomes the subjects will never have to actually experience. Perhaps the observed effects would be stronger or weaker under more realistic conditions. The effect of omitting several, rather than just one, attribute description should be explored, also. Intuitively, the effects ought to be stronger. Are they? Finally, with respect to context effects, it is natural to ask about the influence of the relative frequency of occurrence of partially described options. Are context effects the same when partially described options are rare rather than fairly common?

While the general practical implications of the results are rather apparent, the study highlights some important research issues, also. People are 
often asked by theoretical and applied investigators to evaluate various objects, e.g., cars, schools, proposals, peers, etc. The discrepancy effect results found here suggest that subjects' responses in such studies might reflect systematic biases. Such biases should be taken into consideration when conclusions are drawn from the investigations.

The context effect implicated in the present results forces us to recognize another problem often overlooked in research on judgment, evaluation, and decision processes: One's assessment of the worth of an option is affected not only by what he/she considers to be "important" in the abstract, but by what he/she just happens to attend to when the option is actually presented. Even in as constrained a setting as the standard laboratory displays used in the present study, it appears that subjects' attention to the various aspects of a vailable options can be influenced by rather subtle manipulations. Attention-affecting events in the real world are likely to be more numerous and more powerful. Such naturally occurring attention-affecting events should not be seen as just experimental nuisance factors, however. If one wants to accurately represent how people make real judgments, the effects of such factors must be taken into account. The very tidiness of standard laboratory routines might limit the generality of the representations derived from them. For instance, suppose one wanted to discover the relative significance of various attributes for the attractiveness of a certain kind of product to a given subject. It would be tempting to have the subject identify a few relevant attribute dimensions, present a series of displays of products characterized by those dimensions, and derive a judgment policy equation from his/her responses to those displays. Such a policy equation might be a rather poor representation of how the subject would judge real versions of the product because of attentional considerations. Specifically, while his/her attention to the prescribed dimensions is forced by the nature of the laboratory displays (and reflected in the derived policy equation), he/she might not even notice those characteristics when faced with the actual products. Conversely, features of the products that do, in fact, influence his/her assessments might not do so consciously and, hence, would never have been chosen as display dimensions.

\section{REFERENCES}

Becker, J. W., \& Brownson, F. O. What price ambiguity? Or the role of ambiguity in decision-making. Journal of Political Economy, 1964, 72, 62-73.

Coombs, C. H., Dawes, R. M., \& Tversky, A. Mathematical psychology. Englewood Cliffs, N.J.: Prentice-Hall, 1970.

Ellsberg, D. Risk, ambiguity and the Savage axioms. Quarterly Journal of Economics, 1961, $25,643-669$.

Keeney, R. L., \& Raiffa, H. Decisions with multiple objectives: Preferences and value tradeoffs. New York: Wiley, 1976.

Lee, W. Decision theory and human behavior. New York: Wiley, 1971. 
Luce, R. D., \& Raiffa, H. Games and decisions. New York: Wiley, 1957.

Payne, J. W. Task complexity and contingent processing in decision making: An information search and protocol analysis. Organizational Behavior and Human Performance, 1976, $16,366-387$.

Raiffa, H. Decision analysis. Reading, Mass.: Addison-Wesley, 1968.

Slovic, P., Fischoff, B., \& Lichtenstein, S. Behavioral decision theory. Annual Review of Psychology, 1977, 28, 1-39.

Slovic, P., \& MacPhillamy, D. Dimensional commensurability and cue utilization in comparative judgment. Organiational Behavior and Human Performance, 1974, 11, 172 194.

Summers, D. A., Taliaferro, J. D., \& Fletcher, D. J. Subjective vs. objective description of judgment policy. Psychonomic Science, 1970, 18, 249-250.

Swinth, R. L. A decision process model for predicting job preferences. Jounal of Applied Psychology, 1976, 61, 242-245.

Yates, J. F., \& Zukowski, L. G. Characterization of ambiguity in decision making. Behavioral science, 1976, 21, 19-25.

Received: August 11, 1977 